\title{
Cuidado perdido de enfermería. Estado del arte para la gestión de los servicios de salud
}

\author{
Missing nursing care. State-of-the-art \\ healthcare service management
}

\section{Cuidado perdido de enfermagem. Estado da arte para a gestão dos serviços de saúde}

\author{
R.A. Zárate-Grajales ${ }^{\mathrm{ai}^{*}}$, M. Mejias ${ }^{\mathrm{b} 2}$ \\ ORCID: \\ a $0000-0002-9264-8490$ \\ boooo-0001-6315-1920 \\ ${ }^{1}$ Coordinación de Investigación, Escuela Nacional de Enfermería y Obstetricia, \\ Universidad Nacional Autónoma de México, Ciudad de México, México \\ ${ }^{2}$ Unidad de Investigación en Enfermería (UNIVE), Escuela de Enfermería \\ Dra. Gladys Román de Cisneros, Valencia, Venezuela
}

Recibido: 07 septiembre 2018/Aceptado: 30 junio 2019

\section{RESUMEN}

Introducción: El cuidado perdido de enfermería es un fenómeno recientemente explorado que afecta la seguridad de los pacientes, la calidad de la atención y cuestiona la actuación de los profesionales de enfermería.

Objetivo: Conocer el Estado del Arte del tema Cuidado perdido en Enfermería a partir de artículos publicados durante el periodo marzo 2012- marzo 2018.

Metodología: Se realizó una investigación documental a través del sistema de búsqueda PUBMED, con las siguientes palabras clave: missed nursing care, misscare AND care nurse. Se seleccionaron 47 artículos de investigación en idioma inglés y español. Posterior al análisis de contenido de los artículos, los resultados se agruparon en categorías. 
Resultados: Se identificaron las siguientes categorías: país donde se realizó la investigación, unidades de atención, tipo de estudio, muestra estudiada, instrumento empleado, marco conceptual, así como los elementos, razones y predictores del cuidado perdido.

Conclusiones: El estudio del fenómeno puede ser un insumo para la toma de decisiones en políticas de salud y en el diseño de estrategias e intervenciones que contribuyan a la mejora del cuidado en las instituciones de salud. La revisión del fenómeno y el análisis permitirán a los interesados en el tema, profundizar en el cuidado perdido e identificar la fundamentación teórica, los elementos conceptuales y los resultados encontrados, los cuales serán aportes para la toma de decisiones en los procesos de mejora, la docencia, el cuidado y la investigación. Palabras clave: Enfermería; cuidado perdido; calidad; seguridad del paciente; México.

\section{ABSTRACT}

Introduction: Missing nursing care is a recently explored phenomenon which has impacts on patient security and quality of attention, and which questions the performance of nursing professionals.

Objective: To gain understanding on the phenomenon of missing nursing care from the lens of state-of-the-art care management by reviewing related articles published in the period March 2012 - March 2018.

Methodology: A documentary research was carried out in the PUBMED database using the following key terms: missed nursing care, misscare, AND nurse care. 47 research articles written in English or Spanish were selected. From the content analysis, the results were clustered in categories.

Results: The following categories were selected: country were the research took place, units of attention, type of study, sample studied, instrument used, conceptual frame, and the elements, reasons, and predictors related to missing nursing care.

Conclusions: The study of this phenomenon can bring about bases of orientation to the process of political decision-making, and strengthen the strategies and interventions in the practice, teaching, and research areas which can contribute to provide a better healthcare in institutions.

Keywords: Nursing; missing care; quality; patient security; Mexico.

\section{RESUMO}

Introdução: O cuidado perdido de enfermagem é um fenómeno recentemente explorado que afeta a segurança dos pacientes, a qualidade da atenção e questiona a atuação dos profissionais de enfermagem.

Objetivo: Conhecer o Estado da Arte do tema Cuidado perdido em Enfermagem a partir de artigos publicados durante o período março 2012- março 2018.

Metodologia: Realizou-se uma pesquisa documental a través do sistema de busca PUBMED, com as seguintes palavras chave: missed nursing care, misscare AND care nurse. Escolheramse 47 artigos de pesquisa em idioma inglês e espanhol. Posterior à análise de conteúdo dos artigos, os resultados agruparam-se em categorias.

Resultados: Identificaram-se as seguintes categorias: país onde se realizou a pesquisa, unidades de atenção, tipo de estudo, amostra estudada, instrumento empregado, marco conceitual, assim como os elementos, razões e preditores do cuidado perdido.

Conclusões: O estudo do fenómeno pode ser um insumo para a toma de decisões em políticas de saúde e no desenho de estratégias e intervenções que colaborem para a melhora do 
cuidado nas instituições de saúde. A revisão do fenómeno e a análise permitiram aos interessados no tema, aprofundar no cuidado perdido e identificar a fundamentação teórica, os elementos conceituais e os resultados encontrados, os quais serão contribuições para a tomada de decisões nos processos de melhora, a docência, o cuidado e a pesquisa.

Palavras chave: Enfermagem; cuidado perdido; qualidade; segurança do paciente; México.

\section{INTRODUCCIÓN}

En las instituciones de salud se reconoce que existen algunos riesgos durante la estadía de los pacientes; incluso se tienen reportes de diversos incidentes relacionados con la calidad del cuidado en las instituciones y la seguridad del paciente. Todos los días se notifican quejas relacionadas con la atención que brinda el equipo de salud y las consecuencias negativas que se pueden producir en los pacientes, lo que pone de relieve la importancia de la seguridad de este, que como tal, se constituye en una prioridad de la atención sanitaria; de ahí que sea tema de estudios y de recomendaciones de diversos organismos internacionales, en búsqueda de identificar elementos, factores y diseñar intervenciones que ayuden a disminuir el problema. En octubre de 2004, la Organización Mundial de la Salud (OMS) lanzó oficialmente la Alianza Mundial para la Seguridad del Paciente, en respuesta a la Resolución WHA55.18 adoptada por la 55 Asamblea Mundial de la Salud en mayo de 2002, que exhortaba a los Estados Miembros a prestar atención al tema de la seguridad para mejorar la calidad de la atención sanitaria².

La seguridad del paciente se define como la reducción de riesgos y daños innecesarios relacionados con la atención sanitaria hasta un mínimo aceptable; el cual se refiere a las nociones colectivas de los conocimientos del momento, los recursos disponibles y el contexto en el que se presta la atención, ponderadas frente al riesgo de no dispensar tratamiento o de dispensar otro². La atención sanitaria involucra riesgos para el paciente, aunque el personal sanitario presta el cuidado para promover la salud y el bienestar, pueden surgir situaciones que afecten de manera negativa a este y, en consecuencia, producirle una lesión temporal o permanente e incluso la muerte.

La preocupación por mejorar la calidad de la atención al paciente y sus componentes fundamentales como la seguridad, son algunos de los desafíos que enfrentan los profesionales de enfermería en todo el mundo 3 . A nivel mundial se han reportado errores de los profesionales de la salud y esto involucra el cuidado que brinda enfermería, señalado como una atención negligente, de mala práctica, cuidado deshumanizado, poco ético en el cumplimiento de sus deberes, entre otros aspectos. Se ha identificado que los errores reportados pueden ser resultado de un acto de comisión como aquellos que ejecuta el profesional de enfermería al proporcionar el cuidado, por ejemplo, administrar un medicamento incorrecto, o por un acto de omisión, o sea, aquel cuidado que se deja de realizar, como no administrar el medicamento; ambas situaciones pueden conducir a un resultado adverso4.

Los errores de omisión son más difíciles de reconocer que los errores de comisión, pero es probable que los primeros representen un problema mayor; es decir, se ha documentado en la literatura que hay muchos más casos en los que la provisión de cuidados o intervenciones diversas para el diagnóstico, tratamiento o bien, enfocadas a la prevención, hubieran mejorado la atención, en lugar de casos en los que la atención que necesitaba el paciente no fue proporcionada adecuadamentes.

Por otro lado, en relación con el cuidado, el cuidado perdido de enfermería se considera un error de omisión'; debido a las consecuencias que estos generan en la seguridad y salud de los pacientes. Se han realizado investigaciones para identificar los elementos y razones por las cuales el cuidado de enfermería se omite, retrasa o pierde; como respuesta a la necesidad de comprender el fenómeno 
y los mecanismos que vinculan al personal de enfermería con los resultados en materia de calidad y seguridad.

El cuidado que el personal de enfermería deja sin hacer durante su jornada laboral, es un fenómeno que ha sido estudiado en mayor medida durante la última década. Sochalski ${ }^{6}$, realizó una investigación que demostró que la calidad de la atención de enfermería se asoció significativamente con la cantidad de pacientes atendidos por este personal, las tareas no finalizadas y la frecuencia de los problemas relacionados con la seguridad del paciente. En dicho estudio se empleó el término tareas no finalizadas (tasks undone), para referirse a aquellas actividades del cuidado que enfermería no finalizaba por la carga de trabajo, las cuales podrían ser un factor asociado a los problemas de seguridad del paciente en los hospitales; sin embargo, también se destaca que otros factores como el entorno laboral, juegan un papel importante y podrían interferir con los esfuerzos de enfermería para mejorar la calidad del cuidado.

Al respecto, Schubert7 menciona y acuña el concepto de racionamiento del cuidado de enfermería (Rationing of nursing care) como la retención o falta de tiempo para realizar las tareas de enfermería necesarias para los pacientes, así como el número de personal y la interacción de las habilidades del personal. Menciona el autor que los atributos de la organización del hospital y el entorno de la práctica de enfermería, pueden influir en las decisiones que el personal toma para racionar el cuidado.

El impacto que las tareas dejadas de hacer o la racionalización del cuidado tienen en la seguridad del paciente, fue motivo para que Kalisch ${ }^{8}$, posterior a varias investigaciones, desarrollara el concepto de cuidado perdido de enfermería (missed nursing care, MNC por sus siglas en inglés) que conceptualiza como cualquier aspecto de la atención que requiere el paciente y que se omite (en parte o en su totalidad) o se retrasa.

Al respecto, Ausserhofer ${ }^{9}$ empleó el término cuidado de enfermería dejado de lado (nursing care left undone) para referirse a las actividades de enfermería necesarias para los pacientes, pero que se perdieron por falta de tiempo. También describe cómo los factores relacionados con la organización del entorno de trabajo de enfermería, pueden influir en el proceso de cuidado que incluye la toma de decisiones de dejar ciertas tareas sin realizar.

Los diferentes elementos conceptuales utilizados para describir las tareas o actividades del cuidado de enfermería que se dejan sin realizar, reflejan el interés de los investigadores por comprender el fenómeno del cuidado perdido, las tareas no finalizadas o dejadas de lado que se omiten parcial o totalmente, y los factores que inciden para que el fenómeno se produzca. La comprensión del fenómeno ayuda a los tomadores de decisiones en materia de políticas de salud, a diseñar intervenciones que contribuyan a mitigar este fenómeno que afecta la calidad de la atención y la seguridad del paciente.

\section{Cuidado perdido en enfermería: Implicaciones}

Los investigadores, los hospitales y el propio sistema de salud en algunos países, han volteado la mirada a fin de reconocer los casos de omisión y profundizar en el estudio del cuidado perdido. Investigaciones consultadas muestran que si esto no es identificado y abordado de manera temprana por los responsables de las políticas de salud, podría contribuir a resultados negativos en el cuidado del paciente, así como disminuir los niveles de seguridad y calidad del cuidado; también puede tener implicaciones financieras en términos de mayores costos de atención sanitaria debido al incremento en la estancia hospitalaria, al igual que re-admisiones para el tratamiento de complicaciones y eventos adversos de la atención. La literatura reporta como un factor para el cuidado perdido 
la constante rotación del personal de enfermería en turno de guardia y servicio de atención ${ }^{10}$; lo que influye en el incremento de los costos sociales. Esta situación, a decir de los estudiosos del tema, genera un círculo vicioso en donde los encargados de formular políticas de salud reducen los recursos para contratar personal de enfermería, lo que crea la falsa ilusión de ahorros financieros ${ }^{11}$.

Por otro lado, se ha identificado que los estudios relacionados con la seguridad del paciente vinculados con el personal de enfermería se abordan con mayor frecuencia a partir del error de comisión que de omisión. Sin embargo, esta situación se está revirtiendo al observar el alcance que tiene considerar los errores de omisión como un factor clave para garantizar la seguridad del paciente y la importancia que tiene que los tomadores de decisiones identifiquen los factores y realicen ajustes en las políticas de recursos humanos, contrataciones, índices para el cálculo de personal así como, ante el complejo escenario en el sistema de salud; las condiciones de trabajo y, la cronicidad agravada del paciente, aunado a las características que demanda el cuidado de enfermería en este panorama.

Es así como el cuidado perdido de enfermería está siendo estudiando ampliamente a nivel mundial, y México no escapa de esta realidad. Moreno ${ }^{12}$ se refirió al fenómeno como omisión en la atención de enfermería, cuyo estudio se sustenta en el marco conceptual del modelo de Kalisch de cuidado perdido de enfermería; con el que obtuvo resultados de investigación que le permitieron identificar a través de la percepción del personal de enfermería y del paciente, que existen cuidados que no se realizan de acuerdo con las necesidades específicas del paciente durante su estancia hospitalaria; mismos que en algunas ocasiones son omitidos o retrasados, lo que puede afectar los resultados globales de la atención.

Por su parte, en su estudio Hernández R. ${ }^{13}$ concluyó que las intervenciones de cuidado básico: planificación del alta y educación del paciente, son en las que se presenta mayor omisión; ambas representan cuidados independientes y propios de enfermería que no se deberían de perder u omitir durante la estancia hospitalaria de los pacientes. A su vez, Zárate-Grajales et al. ${ }^{14}$ concluyeron que los pacientes pueden sufrir eventos adversos durante la estancia hospitalaria y que estos pueden ser multifactoriales, en su reporte se observó que ningún evento ocurrió por una determinante aislada, en todos ellos estuvieron presentes los factores intrínsecos, los extrínsecos y los del sistema, en mayor o menor grado. De ahí la necesidad de profundizar en el estudio de eventos adversos reportados en los hospitales y su relación con la omisión del cuidado, ya que los autores identifican que un porcentaje considerable de eventos adversos está relacionado con situaciones del cuidado que han sido reportadas por el personal de enfermería.

\section{METODOLOGÍA}

Se llevó a cabo una revisión documental para conocer el Estado del Arte del tema Cuidado perdido en enfermería. Se ubicaron artículos de investigación a través del sistema de búsqueda PUBMED, con las palabras: missed nursing care, misscare AND nurse care; se consideraron los artículos publicados en el periodo marzo 2012 a marzo 2018. Para la selección de los artículos de investigación se consideró que las palabras de búsqueda estuvieran presentes en el título de los artículos, el idioma español o inglés, y que fuesen artículos de investigación a texto completo. Los resultados obtenidos con las palabras clave: missed nursing care fueron 1113 artículos de los cuales se seleccionaron 61 artículos cuyos títulos contenían missed nursing care. Para la búsqueda con las palabras: misscare AND nurse care se encontraron 24 artículos, de los cuales se seleccionaron dos artículos que contenían en el titulo las palabras de búsqueda. 
Se identificaron un total de 63 resúmenes de artículos seleccionados. Al leer los textos completos de los artículos se descartaron: revisión sistemática (tres), boletín (dos), estudio de caso (uno), editoriales (tres), comentarios (dos) y artículos que no tenían acceso libre (cinco). Se seleccionaron 47 artículos de investigación en idioma inglés o español. Se identificó en la revisión de los artículos seleccionados aspectos clave del cuidado perdido que se organizaron en las siguientes categorías: país donde se realizó la investigación, unidades de atención, tipo de estudio, muestra estudiada, instrumento empleado, marco conceptual, elementos, razones y predictores del cuidado perdido.

\section{RESULTADOS}

La revisión de los artículos del cuidado perdido de enfermería, publicados a texto completo entre el periodo marzo del 2012 a marzo 2018, permitió identificar las vías más comunes que los investigadores emplean para abordar el fenómeno del cuidado perdido y plantear dimensiones acerca de los aspectos considerados en el estudio del tema, los cuales se presentan en tablas anexas.

Del análisis del tipo de estudio y la metodología, se identificó que 38 artículos (81 \%) utilizaron la metodología cuantitativa; de los cuales $66 \%$ son de corte descriptivo transversal, $12.8 \%$ correlacional y $2.1 \%$ cuasi experimental. Los nueve artículos restantes (19\%) emplearon metodología cualitativa, de estos $12.8 \%$ representan análisis de textos, $4.3 \%$ grupo focal y $2.1 \%$ etnográfico.

Entre los paises que han explorado el fenómeno del cuidado perdido, figuran Estados Unidos (48.9\%), seguido por la República de Islandia (4.3\%), Australia (4.3\%), Corea del Sur (4.3\%) y México (4.3\%).El resto de los países como Reino Unido, Jordania, Islandia, Nueva Zelandia, Brasil, Suecia, Israel, República de Chipre, Suiza, Irán y Canadá; se reporta un estudio en cada uno de ellos. Asimismo, el impacto del fenómeno en la calidad del cuidado y en el sistema de salud, ha motivado a los investigadores a realizar estudios conjuntos en diferentes países; encontramos resultados de investigaciones entre Australia, Chipre e Italia; Australia y Nueva Zelandia; EE.UU y Líbano; Nueva Zelandia y Australia; Bélgica, Inglaterra, Finlandia, Irlanda, Países Bajos, Noruega, España, Suecia y Suiza. En cada grupo de países se ha reportado al menos un trabajo de investigación.

Las unidades de atención en donde se realizó el estudio fueron las áreas médico quirúrgicas (45\%); cuidados intensivos (23\%); rehabilitación (11\%); obstetricia, emergencia y asilo de ancianos con un $5 \%$ cada una y UCI neonatal y pediatría (3\%); cabe señalar que en algunos estudios no se específica el tipo de unidad explorada ya que su propósito fue medir el cuidado perdido global y no por unidad de atención. Las unidades menos estudiadas son las pediátricas, psiquiátricas, urgencias, neonatales y obstétricas.

Con relación a la población estudiada, $69 \%$ corresponde a personal de enfermería, $29 \%$ pacientes, y $2 \%$ estudiantes de enfermería. Los resultados evidencian el interés de los investigadores de incorporar al análisis del fenómeno a los pacientes como elementos que reciben el cuidado y cuyas consecuencias repercuten en su salud; y a los estudiantes de enfermería como agentes que comparten el cuidado en las áreas hospitalarias que son escenarios reales de aprendizaje, en los cuales se pierde u omite el objeto de la disciplina.

Los estudios referidos permitieron a los investigadores construir un índice de cuidado perdido de enfermería que se calculó al dividir la cantidad total de ocurrencias de cuidado perdido de enfermería entre el número total de respuestas de la encuesta que no indicó cuidado perdido. Las unidades con los índices más altos de cuidado perdido fueron: renal (1.4), posquirúrgica aguda (1.0), cardíaca (1.0) y ortopedia / neurológica (o.9). Los valores altos indican una mayor cantidad de cuidado perdido, mientras que los valores más bajos indican un menor cuidado perdido ${ }^{15}$. Si bien existen reportes 
de que la complejidad del entorno clínico puede llevar al personal de enfermería a racionar las actividades del cuidado del paciente; la construcción y validación de un índice de cuidado perdido de enfermería podría ayudar a las organizaciones a identificar la incidencia del cuidado perdido, además de guiar los esfuerzos para los programas de mejora. No obstante, se necesitan más investigaciones para desarrollar evaluación comparativa entre organizaciones ${ }^{15}$.

Asimismo, en esta revisión se identificaron los instrumentos empleados para investigar los fenómenos de interés alrededor del cuidado perdido, entre los cuales se encuentran: la Evaluación del Consumidor Hospitalario de Proveedores y Sistemas de Salud (HCAHPS, por sus siglas en inglés) ${ }^{16,17}$. El uso de la encuesta HCAHPS es un enfoque novedoso para cuantificar la prevalencia de la atención olvidada de enfermería en general y por tipo. A través de la encuesta HCAHPS se intenta desarrollar un índice que represente la eficacia de los servicios de enfermería a nivel de unidad, hospital o sistema ${ }^{15}$. La encuesta está dirigida al paciente que estuvo hospitalizado por lo menos 24 horas, en la que se evalúan aspectos relacionados con la atención brindada por médicos y enfermeras, ambiente hospitalario, experiencia del paciente, egreso, clasificación del hospital y datos del paciente.

Otro grupo de investigadores han relacionado al cuidado perdido de enfermería con el ambiente laboral, para esto utilizaron la Escala de Entorno de la Práctica Enfermera del Nursing Work Index (PES-NWI, por sus siglas en inglés) ${ }^{18,19}$, un instrumento que consta de cinco dominios, 31 ítems con un puntaje tipo Likert de cuatro puntos (que van desde muy en desacuerdo hasta muy de acuerdo), que pide a las enfermeras que caractericen su ambiente de trabajo ${ }^{18}$.

En esa misma línea, Bragadóttir et al. ${ }^{20}$ usaron en su investigación la combinación de dos encuestas para obtener datos sobre el MNC y el trabajo en equipo de enfermería, estos fueron la Encuesta de MISSCARE-Islandés y la Encuesta de Trabajo en Equipo de Enfermería-Islandés (NTS-Icelandic, por sus siglas en inglés). La encuesta NTC-Icelandic es la traducción de la versión estadounidense de la Encuesta de Trabajo en Equipo de Enfermería (NTS) publicada por Kalisch et al., y consta de 33 ítems divididos en cinco subescalas, las cuales surgieron de los datos: 1. Confianza (siete ítems), 2. Orientación del equipo (nueve ítems), 3. Respaldo/apoyo (seis ítems), 4. Modelo mental compartido (siete ítems) y 5 . Liderazgo del equipo (cuatro ítems) ${ }^{20}$.

El marco conceptual de las investigaciones analizadas en su gran mayoría (67.9\%), se sustentan en el Modelo de Cuidado Perdido propuesto por Kalisch (Tabla 1). El concepto de cuidado perdido de enfermería fue desarrollado para mostrar los diversos atributos de las categorías reportadas por el personal de enfermería que contribuyen al cuidado perdido. Los atributos incluyen: a. Antecedentes dentro del entorno de cuidado que facilitan o no la práctica de la enfermería (recursos laborales, recursos materiales, relaciones y comunicación); b. Elementos del proceso de enfermería; c. Percepciones de la enfermera(o) y procesos de decisión (por ejemplo: valores, creencias, hábitos, etc.); d. La atención que se brinda según lo planificado y la atención demorada u omitida (cuidado perdido), y por último; e. Resultados del paciente4.

El tema del cuidado perdido de enfermería se constituye en una enorme controversia si se analiza sólo a partir de las actividades que el personal de enfermería reporta como no realizadas, omitidas o retrasadas durante su jornada laboral. Los estudios reportan los aspectos del cuidado que con mayor frecuencia se omiten ${ }^{3,15,18,5,17}$ entre los que se encuentran: el cuidado de la boca; baño del paciente y cuidado dela piel, apoyo en la deambulación; alimentación del paciente; administración de medicamentos prescritos; educación del paciente; respuesta a la llamada dentro de los 5 min.; comunicación; control de los fluidos parenterales y de niveles de glucosa en sangre; asistencia después del alta (egreso); evaluación de los signos vitales; brindar apoyo emocional al paciente y a la familia; y planes de atención (Tabla 2). 
Tabla 1. Marco conceptual que sustentan las investigaciones acerca del cuidado perdido

\begin{tabular}{|c|c|c|}
\hline Marco conceptual o base teórica & $\mathbf{N}^{\circ}$ & $\%$ \\
\hline Modelo de cuidado perdido en enfermería (Kalisch, 2009): 3, 9, 10, 12, 13,18-50 & 38 & 67.9 \\
\hline Modelo de cuidado perdido: valores y creencias (Kalisch, 2009) ${ }^{51}$ & 1 & 1.8 \\
\hline Fundamentos del cuidado desarrollado por Kitson et al., 2010; Kitson, Conroy et al., $2013^{52}$ & 1 & 1.8 \\
\hline $\begin{array}{l}\text { Clasificación de la atención sobre la base de la percepción de la enfermera de las necesidades } \\
\text { inmediatas del paciente (Lefevre, 2011) }\end{array}$ & 1 & 1.8 \\
\hline Reformas en el sector salud 53 & 1 & 1.8 \\
\hline Modelo de Climas éticos (Victor B and Cullen JB., 1987)54 & 1 & 1.8 \\
\hline Teorías de liderazgo. Teoría de la congruencia (Cogliser et al. 2009)55 & 1 & 1.8 \\
\hline Modelo de trabajo en equipo (Salas, Sims y Burke, 2005) ${ }^{56}$ & 1 & 1.8 \\
\hline Carga de trabajo de enfermería en UCI (Cacayon y Gursts, 2005)57 & 1 & 1.8 \\
\hline Modelo de organización y resultado (Aiken et al. 2002)39 & 1 & 1.8 \\
\hline Razonamiento implícito del cuidado de enfermería (Schubert et al. 2007) 58 $^{8}$ & 1 & 1.8 \\
\hline Encuesta HCAHPS ${ }^{15}$ & 1 & 1.8 \\
\hline $\begin{array}{l}\text { Término cuidado perdido: En este grupo se incluyen investigaciones cuyos autores utilizan el } \\
\text { término de cuidado perdido, tareas no finalizadas 9, 16,20,50,59-61 }\end{array}$ & 7 & 12.3 \\
\hline
\end{tabular}

Los elementos mencionados constituyen aspectos básicos del cuidado que brinda enfermería, cuya omisión influye en el cuidado del paciente y su seguridad; pareciera contradictorio pensar que los cuidados básicos se omiten cuando los profesionales de enfermería conocen la importancia de estos para la satisfacción de las necesidades del paciente. Sin embargo, parte del cuidado que se omite se puede atribuir al cuidado invisible, ya que no se registra o documenta lo que realiza enfermería al monitorizar a los pacientes, al educar a pacientes y familias, al elaborar los planes de alta, al proporcionar apoyo psicológico a los pacientes y sus familiares. Lo que muestra la literatura revisada es el trabajo más visible de este personal que se orienta a las tareas interdependientes o dependientes, como tomar signos vitales, administrar medicación, ayudar a los pacientes a comer, deambular, ir al baño, etc. ${ }^{11}$.

Son estos elementos del cuidado perdido de enfermería, colectivos o independientes, que pueden dar paso a la presencia de error por omisión o llegar a producir un evento adverso en el paciente ${ }^{49}$. Ante estos hechos reportados en la literatura, se podría pensar que los profesionales de enfermería no cumplen con los cuidados requeridos por los pacientes en las unidades hospitalarias, sin embargo, existen investigaciones que han demostrado la existencia de múltiples factores que influyen en el cuidado perdido, entre ellos las características de los recursos humanos, dotación de insumos, la gravedad del paciente y otras que se han estudiado y reportado. Más aún, en el cuidado perdido de enfermería se reportan diversos factores, elementos o circunstancias que contribuyen a que dicha situación se genere, lo que afecta el desempeño de los profesionales y en consecuencia la seguridad del paciente.

Los resultados de las investigaciones integradas en esta revisión, revelan la existencia de factores que influyen en el cuidado perdido de enfermería (Tabla 3), entre los cuales se mencionan:

- La organización de la institución de salud, del hospital o los servicios hospitalarios que constituyen uno de los principales factores reportados por el personal de enfermería para la omisión del cuidado ${ }^{52}$. 
Tabla 2. Elementos del cuidado perdido

\begin{tabular}{|c|c|}
\hline $\begin{array}{l}\text { Elementos del cuidado } \\
\text { perdido }\end{array}$ & Artículos \\
\hline Cuidado de la boca & $\begin{array}{l}\text { Cuidado de la boca51, cuidado de la boca }(35.7 \%)^{49} \text {. Cuidado de la boca } \\
(74 \% \text { y } 59 \%)^{38} \text {. Cuidado bucal }(62 \%, n=192)^{37} \text {. }\end{array}$ \\
\hline $\begin{array}{l}\text { Baño del paciente / cuidado } \\
\text { de la piel }\end{array}$ & $\begin{array}{l}\text { Uso del baño (10.1\%). Baño de pacientes / cuidado de la piel }{ }^{49} \text {. Baño de } \\
\text { pacientes }{ }^{3} \text {. Baño de paciente }(75.2 \%)^{56} \text {. Cuidado de la piel/cuidado de } \\
\text { heridas (38.5\%). Uso del baño }(64 \% \text { y } 52 \%)^{38} \text {. Cambios de posición del } \\
\text { paciente cada } 2 \text { horas }(73 \%, n=193)^{37} \text {. }\end{array}$ \\
\hline Deambulación & $\begin{array}{l}\text { Deambulación tres veces al día o según lo ordenado }(53 \%)^{49} \text {. } \\
\text { Asistencia con la deambulación ( } 79 \% \text { y } 73 \% \text { para UCSF-P y UCLA-SM, } \\
\text { respectivamente) })^{38} \text {. }\end{array}$ \\
\hline Alimentación del paciente & $\begin{array}{l}\text { Alimentación del paciente, comida caliente }{ }^{51} \text {. Alimentar a los pacientes } \\
\text { mientras sus alimentos estaban calientes }{ }^{3} \text {. Alimentación }(68 \% \text { y } 52 \%)^{38} \text {. } \\
\text { Alimentando al paciente cuando la comida todavía está caliente }(60.5 \% \text {, } \\
\mathrm{n}=165)^{37} \text {. }\end{array}$ \\
\hline $\begin{array}{l}\text { Administración de } \\
\text { medicamentos prescritos }\end{array}$ & $\begin{array}{l}\text { Medicación }(24.4 \%)^{15} \text {. Administración de medicamentos dentro de } \\
\text { los } 30 \text { minutos del programa del tiempo }(31.6 \%)^{49} \text {. Administrando } \\
\text { medicamentos dentro de los } 30 \text { minutos antes o después del horario } \\
\text { programado }(67 \%, \mathrm{n}=176)^{37} \text {. }\end{array}$ \\
\hline Educación del paciente & $\begin{array}{l}\text { La educación del paciente, incluida la explicación de los efectos adversos } \\
\text { de la medicación, la asistencia y la instrucción post-egreso, a menudo } \\
\text { se pierde }{ }^{15} \text {. Educación al paciente }{ }^{3} \text {. Alta del paciente y educación (ambos } \\
\text { con } 31.1 \%)^{56} \text {. }\end{array}$ \\
\hline $\begin{array}{l}\text { Respuesta a la luz de llamada } \\
\text { dentro de los primeros } 5 \text { min }\end{array}$ & $\begin{array}{l}\text { Asistencia telefónica directa }(16.4 \%)^{15} \text {. La respuesta a la luz de llamada } \\
\text { dentro de los } 5 \text { min }^{51} \text {. }\end{array}$ \\
\hline Comunicación & Comunicación perdida (2.33 vs. 1.87) ${ }^{26}$ \\
\hline $\begin{array}{l}\text { Control de los fluidos } \\
\text { parenterales y los niveles de } \\
\text { glucosa en sangre }\end{array}$ & $\begin{array}{l}\text { La ingesta y el rendimiento de líquidos } 3 \text {. La glucosa en sangre según lo } \\
\text { ordenado }(81.6 \%)^{49} \text {. Control de la entrada y salida }(70 \% \text { y } 48 \%)^{38} \text {. }\end{array}$ \\
\hline
\end{tabular}

Asistencia después del alta (egreso)

Asistencia posterior al alta (21.5\%) $)^{15}$. Ayuda durante el alta ${ }^{3}$.

Brinda apoyo emocional al paciente y a la familia

Apoyo emocional para el paciente y la familia 3 .

Planes de atención

Establece el plan de cuidados y lo ejecuta de acuerdo al riesgo de UPP $(72.0)^{56}$

Tabla 3. Factores que influyen en el cuidado perdido

\begin{tabular}{|c|c|}
\hline Factores & $\%$ \\
\hline Organización & $62.7 \%$ la falta de apoyo administrativo ${ }^{42}$ \\
\hline $\begin{array}{l}\text { Recursos } \\
\text { humanos }\end{array}$ & $\begin{array}{l}\text { Niveles de personal inadecuados }(61.1 \%)^{42} \text {. Asistentes inadecuados } 49 \text {. Cantidad } \\
\text { inadecuada de personal }(84.9 \%, \mathrm{n}=192) \text {. Personal inadecuado }(58.9 \%) \text {. Los informes de } \\
\text { horas extras aumentaron a medida que disminuyó el personal } \\
(\mathrm{r}=-.255 ; \mathrm{p}=.001)^{37} \text {. Recursos Humanos } 85.6 \%{ }^{56} \text {. }\end{array}$ \\
\hline Pacientes & $\begin{array}{l}\text { Aumento inesperado en el número y / o la gravedad de los pacientes }(59.9 \%)^{42} \text {. } \\
\text { Aumento inesperado en el volumen / calidad (76.2\%). altas admisiones / egreso }(72 \%) \text {. } \\
\text { situaciones urgentes }(53.0 \%)^{49} \text {. Aumento inesperado del volumen y / o la agudeza del } \\
\text { paciente en la unidad }(87.4 \%, n=190) \text {. Alta actividad de admisión y alta }(80.9 \%, n= \\
189)^{37} \text {. }\end{array}$ \\
\hline $\begin{array}{l}\text { Recursos } \\
\text { materiales }\end{array}$ & $\begin{array}{l}\text { Medicamentos no disponibles cuando se necesitan }(56.5 \%)^{49} \text {. Recursos Materiales } 82.4 \% \\
{ }^{56} \text {. Medicamentos no disponibles cuando es necesario }(73.4 \%, n=177)^{37} \text {. }\end{array}$ \\
\hline Comunicación & $\begin{array}{l}\text { Mala comunicación entre equipo, departamentos auxiliares y personal médico }{ }^{49} \text {. } \\
\text { Comunicación } 81.2 \%{ }^{56} \text {. }\end{array}$ \\
\hline
\end{tabular}


Los problemas de comunicación entre el equipo 49,11, 24 se reportan como elementos principales que configuran el escenario para retrasar o perder el cuidado49. Otro factor que contribuye para que esto suceda es la insatisfacción del personal con sus propios equipos de trabajo y el hecho de que no desean permanecer en su trabajo actual ${ }^{11}$. Diversas investigaciones destacan que de dos a tres de las 15 tareas de cuidado que realiza enfermería se pierden por problemas de comunicación ${ }^{21}$. Este hecho es de vital relevancia al considerar que el quiebre en la comunicación es mencionado en más del 70\% de los eventos centinelas (daños graves a los pacientes), lo que supera a otros factores de riesgo tales como la pobre capacitación técnica de los profesionales de la salud, la insuficiente evaluación del paciente, y la falta del personal necesario para cumplir con la tarea34. Tanto la comunicación como la cooperación rápida entre el personal médico son esenciales para garantizar la seguridad y la atención efectiva 49 .

- Otro factor es el inadecuado perfil del persona ${ }^{499,41,11}$, aunado a los métodos ineficaces para determinar esto ${ }^{42}$ y el racionamiento en la contratación de personal de enfermería como consecuencia de políticas administrativas y de presupuesto. Los hallazgos muestran que el presupuesto del hospital es un factor importante para determinar la adecuación del personal ${ }^{42}$, por lo tanto, los problemas de recursos humanos se identificaron en algunos estudios como la razón más importante para la omisión de la atención (90.9\%) ${ }^{40}$.

A decir del personal de enfermería, otros factores que tienen influencia sobre la atención que se pierde, se explican por la relación entre provisión de recursos y la comunicación entre profesionales de la salud. El personal que percibe una asignación inadecuada de recursos para la atención considera que influye en las tensiones de comunicación entre el personal médico ${ }^{11}$.

- Se ha documentado que ante situaciones financieras críticas que viven los países, visible en la contención de costos, incorporar mayor número de personal adicional a las instituciones de salud puede no ser siempre factible, por lo que se han preocupado por replantear nuevos enfoques para la prestación de cuidados de enfermería, al utilizar el mínimo de personal de manera más eficiente ${ }^{49}$.

En diversas investigaciones el personal de enfermería reporta que tienen demasiados pacientes que cuidar, no tienen tiempo para prestar toda la atención que ellos necesitan, por lo tanto, este cuidado perdido de enfermería aumenta la probabilidad de resultados negativos en los pacientes y mayor riesgo de la seguridad de este al interior del hospital ${ }^{47}$. Así también, se ha reportado que la cantidad y el estado del paciente, el número de actividades relacionadas con admisiones y altas y cambios súbitos en las condiciones del paciente fueron consideradas razones moderadas o importantes para el cuidado perdido. Estos datos sugieren que el sistema de salud es incapaz de responder ante la cantidad de carga de trabajo que existe en las instituciones ${ }^{49}$. Al respecto, mencionan que es el personal quien debe tener las habilidades para adaptarse con el equipo suministrado, a los cambios diarios en la carga de trabajo y al estado del paciente, al igual que el conocimiento para definir prioridades en la atención ${ }^{42}$. Los reportes indican que una cantidad importante de intervenciones de enfermería básicas y clínicamente relevantes para el paciente, se pierden en las unidades médicas donde ingresan pacientes con condiciones clínicas complejas41. 
En los artículos revisados, los investigadores explican que estos factores están relacionados con las políticas de austeridad del sistema de salud, resultado de las reformas implementadas desde hace varias décadas que provocan que los profesionales de enfermería tengan que priorizar entre el cuidado como un componente central de su trabajo, o las expectativas y objetivos administrativos de los gerentes. Ante este panorama, enfermería está tratando de aferrarse a la esencia del cuidado profesional, en un entorno complejo ${ }^{16}$. En otros estudios se muestra que la cantidad inadecuada de personal y el gran flujo de ingresos y egresos fueron los factores reportados por el $90 \%$ de los participantes, se destaca la relación desequilibrada entre la necesidad del paciente y la posibilidad real de que el personal garantice el cuidado necesario ${ }^{41}$.

Otro grupo de estudios identificados en esta revisión, están relacionados con propuestas para identificar aquellos elementos que de no corregirse pueden llevar a perpetuar el cuidado perdido en las organizaciones de salud; a dichos elementos se les ha denominado predictores del cuidado perdido. A continuación, se analizarán algunos de ellos.

Se ha encontrado que la poca participación del personal en los equipos de enfermería es un elemento predictivo del cuidado perdido ${ }^{20,35}$. Se ha identificado una relación estadísticamente significativa entre el cuidado perdido de enfermería y el trabajo en equipo de enfermería ${ }^{20}$. Es innegable que se necesita un trabajo en equipo efectivo para proporcionar respuestas oportunas a las necesidades del paciente. El trabajo en equipo en los servicios de enfermería de cuidados agudos es esencial para la seguridad del paciente y la atención de calidad; sin embargo, los profesionales de esta área manifiestan percepciones ambivalentes con respecto a su trabajo, pues si bien el cuidado sigue siendo fundamental para sus valores, tienden a considerarlo insignificante ante otras tareas ${ }^{50}$; por ello, una intervención de capacitación de supervisores y jefes de servicio para mejorar el trabajo en equipo es deseable, factible y promete ser un enfoque eficaz para aumentar el trabajo en equipo y disminuir los eventos de cuidados perdidos en entornos de atención aguda ${ }^{56}$.

Otros de los elementos presentes en los estudios incluidos en esta revisión, están relacionados con la condición clínica del paciente y el tipo de unidad a la cual está asignado. Se identifica que el tipo de unidad se asocia con el cuidado perdido de enfermería, el cual es más alto en unidades médicas y quirúrgicas, y no así en las unidades de cuidados intensivos ${ }^{20}$. Las mejoras en las condiciones de trabajo percibidas por el personal de enfermería, así como el número de pacientes asignado en unidades de pacientes críticos vs unidades de hospitalización se presenta como un factor que reduce las omisiones de atención y mejora los resultados de los pacientes ${ }^{23}$. Los reportes integrados en esta revisión muestran que cuando existe menos personal y estos tienen más pacientes que atender, existe mayor cuidado perdido, por lo que un aumento en el personal se asociaría con una disminución del cuidado perdido de enfermería ${ }^{25}$. Otros reportes refieren que el perfil adecuado del personal de la unidad, el número de pacientes, las características del área y el trabajo en equipo contribuyen significativamente a la variación del cuidado perdido ${ }^{20}$.

\section{CONCLUSIONES}

La presente revisión permitió identificar que existen algunos vacíos en el conocimiento respecto al cuidado perdido, lo que constituye una oportunidad para realizar proyectos que contribuyan a disminuir la brecha y aporten al conocimiento de la gestión, la calidad y seguridad de los pacientes, entre los cuales se encuentran:

- Identificar índices de cuidado perdido a nivel local, regional y nacional que permitan implementar programas de mejora y evaluar los resultados. 
- Realizar análisis del comportamiento entre regiones (local, nacional e internacional) en donde se encuentran ubicadas las instituciones de salud, de los índices de cuidado perdido de enfermería en función de los índices de enfermera(o) - paciente que prevalecen; lo cual permitirá revisar la normatividad existente respecto a estos indicadores de acuerdo al nivel de complejidad del cuidado requerido en las unidades de atención.

- Valorar el cuidado perdido de enfermería en unidades de atención poco exploradas como son las pediátricas y obstétricas, lo que ayuda a identificar los factores asociados al cuidado perdido para grupos poblacionales de riesgo.

- Llevar a cabo estudios comparativos entre hospitales que permitan medir los elementos y razones de la omisión del cuidado.

- Implementar estudios de intervención integrados a la formación de estudiantes y profesionales, con énfasis en la práctica reflexiva de sus actividades asistenciales a fin de disminuir la omisión del cuidado.

- Evaluar el impacto del cuidado omitido por los profesionales de enfermería sobre el aprendizaje de los estudiantes en los escenarios clínicos.

El presente trabajo permitió realizar un análisis del estado del conocimiento sobre el cuidado perdido a partir de la revisión de diversas posturas y abordajes metodológicos, lo que posibilita valorar la importancia que el cuidado perdido tiene en la calidad y seguridad de los pacientes. Es por ello que profundizar en el estudio del tema en la población y hospitales de México, contribuirá a generar evidencia para la toma de decisiones en materia de recursos humanos para la salud, en programas educativos y de mejora, así como en el diseño de políticas. Comprender que el cuidado que se omite o se retrasa es multifactorial y que está relacionado en la mayoría de las veces con el número de personal, las condiciones del ambiente laboral y la complejidad en el cuidado de los pacientes.

Se pone en evidencia que en América Latina, sólo Brasil tiene estudios reportados y en México existen pocos estudios relacionados con el tema, a diferencia de los Estados Unidos o Europa, en donde existe preocupación sobre el índice de cuidado perdido reportado en las unidades de atención a la salud, lo que evidencia la necesidad de fomentar en México investigaciones relacionadas al cuidado perdido como problema mundial que afecta la seguridad del paciente. Abordar el tema es sentar las bases científicas y metodológicas para proporcionar cuidados de enfermería con calidad.

Esta revisión sobre el cuidado perdido es un acercamiento al tema y pone en la mesa de discusión aquellos factores que afectan la seguridad del paciente y que involucran a los sistemas de salud, las instituciones educativas, los líderes de enfermería y, aporta a los estudiosos de la calidad y seguridad del paciente una mirada que provoque profundizar en esta problemática.

\section{RESPONSABILIDADES ÉTICAS:}

Protección de personas y animales. Este es considerado un estudio sin riesgo porque exclusivamente se realizó revisión de documentos.

Financiamiento. Este artículo se deriva del proyecto PAPITT IN306419 que recibió financiamiento por medio de la DGAPA UNAM.

Conflicto de intereses. Los autores declaran no tener conflicto de intereses. 


\section{REFERENCIAS}

1. Organización Mundial de la Salud. Seguridad del paciente. Ginebra: OMS; 2008. http://bit.ly/334mJAo

2. World Health Organization. Patient Safety. A World Aliance for Safer Health Care. Marco conceptual de la Clasificación Internacional para la seguridad del paciente. Versión 1.1. Genova: WHO; 2009. http://bit.ly/2IpE7qT

3. Blackman I, Papastavrou E, Palese A, Vryonides S, Henderson J, Willis E. Predicting variations to missed nursing care: A three-nation comparison. J Nurs Manag. 2018; 26(1): 33-41.

https://doi.org/10.1111/jonm.12514

4. Kalisch BJ, Xie B. Errors of Omission: Missed Nursing Care. West J Nurs Res. 2014; 36(7):875-90. https://doi.org/10.1177/0193945914531859

5. Agency of Healthcare Research and Quality (AHRO). PSNet Patient Safety Network: Glossary. Rockville, MD: AHRQ; 2014. http://bit.ly/2AJLVzA

6. Sochalski J. Is More Better? The Relationship Between Nurse Staffing and the Quality of Nursing Care in Hospitals. Medical care. 2004; 42(2 suppl): II67-73. https://doi.org/10.1097/01.mlr.0000109127.76128.aa

7. Schubert M, Glass TR, Clarke SP, Aiken LH, Schaffert-Witvliet B, Sloane DM Rationing of nursing care and its relationship to patient outcomes: the swiss extension of the international hospital outcomes study. Int J Qual Health Care 2008; 2O(4): pp. 227-37.https://doi.org/10.1093/intqhc/mzno17

8. Kalisch BJ, Landstrom GL,Hinshaw AS. Missed nursing care: a concept analysis. J Adv Nurs. 2009. 65(7), 1509-17. https://doi.org/10.1111/j.1365-2648.2009.05027.x

9. Ausserhofer D, Zander B, Busse R, Schubert M, De Geest S, Rafferty AM, et al. Prevalence, patterns and predictors of nursing care left undone in European hospitals: results from the multicountry crosssectional RN4CAST study. BMJ Qual Saf 2014; 23(2):126-35. https://doi.org/10.1136/bmjqs-2013-002318

10. Hamilton P, Willis E, Jones T, McKelvie R, Blackman I, Toffoli L, et al. Evaluating the Performance of a Single-Item, Global, Estimate of Missed Nursing Care. J Nurs Meas. $2017 ; 25$ (1):121-41. https://doi.org/10.1891/1061-3749.25.1.121

11. Sasso L, Bagnasco A, Aleo G, Catania G, Dasso N, Zanin M, et al. Incorporating nursing complexity in reimbursement coding systems: the potential impact on missed care. BMJ Qual Saf. 2017; 26(11): 929-32. http://dx.doi.org/10.1136/bmjqs-2017-006622

12. Moreno-Monsiváis MG, Moreno-Rodríguez C, Interial-Guzmán MG. Omisión en atención de enfermería para pacientes hospitalizados. Aquichan. 2015;15 (3):329-38. https://doi.org/10.5294/aqui.2015.15.3.2

13. Hernández-Cruz R, Moreno-Monsiváis MG, Cheverría-Rivera S, Díaz-Oviedo A. Factors influencing the missed nursing care in patients from a private hospital. Rev Lat Am Enfermagem 2017;25: http://dx.doi.org/10.1590/1518-8345.1227.2877

14. Zárate-Grajales R, Salcedo-Álvarez RA, Olvera-Arreola SS, Hernández-Corral S, Barrientos-Sánchez J, Pérez-López MT, et al. Eventos adversos en pacientes hospitalizados reportados por enfermería: un estudio multicéntrico en México.Enferm. univ. 2017. 14 (4). 277 - 85.

https://doi.org/10.1016/j.reu.2017.08.005

15. Orique SB, Patty CM, Sandidge A, Camarena E, Newsom R. Quantifying Missed Nursing Care Using the Hospital Consumer Assessment of Healthcare Providers and Systems (HCAHPS) Survey. J Nurs Adm. 2017; 47 (12):616-22. https://doi.org/10.1097/NNA.0000000000000556

16. Ball JE, Bruyneel L, Aiken LH, Sermeus W, Sloane DM, Rafferty AM, et al. Post-operative mortality, missed care and nurse staffing in nine countries: A cross-sectional study. Int J Nurs Stud. 2018;78:10-15. https://doi.org/10.1016/j.jinurstu.2017.08.004 
17. Lake ET, Germack HD, Viscardi MK. Missed nursing care is linked to patient satisfaction: a cross-sectional study of US hospitals. BMJ Qual Saf. 2016; 25(7): 535-43. http://doi.org/10.1136 /bmjqs-2015-003961

18. Hessels AJ, Flynn L, Cimiotti JP, Cadmus E, Gershon R. The impact of the nursing practice environment on missed nursing care. Clin Nurs Stud. 2015; 3, (4): 6o-5. https://doi.org/10.5430/cns.v3n4p6o

19. Lake ET, de Cordova PB, Barton S, Singh S, Agosto PD, Ely B, et al. Missed nursing care in pediatrics. Hosp Pediatr. 2017; 7(7): 378-84. https://doi.org/10.1542/hpeds.2016-0141

20. Bragadóttir H, Kalisch BJ, Tryggvadóttir GB. Correlates and predictors of missed nursing care in hospitals. J Clin Nurs. 2017; 26 (11-12):1524-34. http://doi.org/10.1111/jocn.13449

21. Blackman I, Henderson J, Willis E, Hamilton P, Toffoli L, Verrall C, et al. Factors influencing why nursing care is missed. J Clin Nurs. 2015; 24 (1-2):47-56. https://doi.org/10.1111/jocn.12688

22. Bragadóttir H, Kalisch BJ. Comparison of reports of missed nursing care: Registered Nurses vs. practical nurses in hospitals. Scand J Caring Sci. 2018; 32(3):1227-36. https://doi.org/10.1111/scs.12570

23. Carthon JM, Lasater KB, Sloane DM, Kutney-Lee A. The quality of hospital work environments and missed nursing care is linked to heart failure readmissions: a cross-sectional study of US hospitals. BMJ Qual Saf. 2015; 24 (4): 255-63. https://doi.org/10.1136/bmjqs-2014-003346

24. Castner J, Wu YW, Dean-Baar S. Multi-Level Model of Missed Nursing Care in the Context of Hospital Merger. West J Nurs Res. 2015; 37 (4): 441-61. https://doi.org/10.1177/0193945914535670

25. Cho SH, Kim YS, Yeon KN, You SJ, Lee ID. Effects of increasing nurse staffing on missed nursing care. Int Nurs Rev. 2015 Jun; 62 (2): 267-74. https://doi.org/10.1111/inr.12173

26. Cho SH, Mark BA, Knafl G, Chang HE, Yoon HJ. Relationships Between Nurse Staffing and Patients' Experiences, and the Mediating Effects of Missed Nursing Care. J Nurs Scholarsh. 2017; 49 (3):347-55. https://doi.org/10.1111/jnu.12292

27. Dabney BW, Kalisch BJ. Nurse staffing levels and patient-reported missed nursing care. J Nurs Care Qual. 2015;30(4):306-12. https://doi.org/10.1097/NCQ.0000000000000123

28. Siqueira-Dias Castilho L, Caliri-Larcher MH, Haas Vanderlei J, Kalisch B, Dantas-Spadoti RA. Validation of the MISSCARE-BRASIL survey-A tool to assess missed nursing care. Rev lat-am enferm. 2017; 25. http://dx.doi.org/10.1590/1518-8345.2354.2975

29. Friese RC, Kalisch BJ, Lee KH. Patterns and correlates of missed nursing care in inpatient oncology units. Cancer nurs. 2013; 36(6): E51-7. https://doi.org/10.1097/NCC.obo13e318275f552

30. Gibbon B, Crane J. The impact of 'missed care'on the professional socialisation of nursing students: A qualitative research study. Nurse Educ Today 2018; 66:19-24. https://doi.org/10.1016/j.nedt.2018.04.002

31. Kalisch BJ, Gosselin K, Choi SH. A comparison of patient care units with high versus low levels of missed nursing care. Health care management review, 2012; 37(4): 320-8.

https://doi.org/10.1097/HMR.obo13e318249727e

32. Kalisch BJ, Lee KH. Missed nursing care: Magnet versus non-Magnet hospitals. Nurs outlook 2012; 6o(5); e32-e39. https://doi.org/10.1016/j.outlook.2012.04.006

33. Kalisch BJ, Tschannen D, Lee KH. Missed nursing care, staffing, and patient falls. J Nurs Care Qual. 2012; 27(1):6-12. https://doi.org/10.1097/NCQ.obo13e318225aa23

34. Kalisch BJ,Doumit M, Lee KH, Zein JE. Missed nursing care, level of staffing, and job satisfaction:Lebanon versus the United States.J Nurs Adm. 2013; 43(5):274-9. https://doi.org/10.1097/NNA.obo13e31828eebaa

35. Kalisch BJ, XIE B, Dabney BW. Patient-reported missed nursing care correlated with adverse events. Am J Med Qual. 2014; 29(5): 415-22. https://doi.org/10.1177/1062860613501715 
36. Knopp-Sihota JA, Niehaus L, Squires JE, Norton PG, Estabrooks CA. Factors associated with rushed and missed resident care in western Canadian nursing homes: a cross-sectional survey of health care aides. J Clin Nurs 2015; 24(19-20): 2815-25. https://doi.org/10.1111/jocn.12887

37. Maloney S, Fend JL, Hardin SR. Is Nursing Care Missed? A Comparative Study of Three North Carolina Hospitals. Med Surg Nurs. 2015; 24(4): 229-35.

38. McNair N, Baird J, Grogan TR, Walsh CM, Liang LJ. Worobel-Luk P, et al. Is Spending More Time Associated With Less Missed Care?: A Comparison of Time Use and Missed Care Across 15 Nursing Units at 2 Hospitals. J Nurs Adm. 2016;46(9):428-37.https://doi.org/10.1097/NNA.0000000000000371

39. Nelson ST, Flynn L. Relationship between missed care and urinary tract infections in nursing homes. Geriatr Nurs. 2015; 36(2):126-30. https://doi.org/10.1016/j.gerinurse.2014.12.009

40. Orique SB, Patty CM, Woods E. Missed Nursing Care and Unit-Level Nurse Workload in the Acute and Post-Acute Settings. J Nurs Care O-ual. 2016; 31 (1):84-9.

https://doi.org/10.1097/NCQ.0000000000000140

41. Palese A, Ambrosi E, Prosperi L, Guarnier A, Barelli, Pet al. Missed nursing care and predicting factors in the Italian medical care setting. Intern Emerg Med. 2015; 10 (6):693-702.

https://doi.org/10.1007/s11739-015-1232-6

42. Phelan A, McCarthy S, Adams E. Examining missed care in community nursing: A cross section survey design. J Adv Nurs. 2018;74 (3):626-36. https://doi.org/10.1111/jan.13466

43. Saqer TJ, Abualrub RF. Missed nursing care and its relationship with confidence in delegation among hospital nurses. J Clin Nurs. 2018; 27(13-14): 2887-95. https://doi.org/10.1111/jocn.14380

44. Simpson KR, Lyndon A. Consequences of delayed, unfinished, or missed nursing care during labor and birth. J Perinat Neonatal Nurs. 2017; 31(1):32-40. http://doi.org/10.1097/JPN.0000000000000203

45. Tubbs-Cooley HL, Pickler RH, Mara CA, Othman M, Kovacs A, Mark BA. Hospital Magnet ${ }^{\circledR}$ designation $^{-}$ and missed nursing care in neonatal intensive care units. J Pediatr Nurs. 2017; 34: 5-9.

https://doi.org/10.1016/j.pedn.2016.12.004

46. Hernández-Valles JH, Moreno-Monsivais MG, Interial-Guzman MG, Vázquez-Arreola L. Nursing care missed in patients at risk of or having pressure ulcers. Rev. Lat-am Enfermagem 2016; 24. http://dx.doi.org/10.1590/1518-8345.1462.2817

47. Verrall C, Abery E, Harvey C, Henderson J, Willis E, Hamilton P, et al. Nurses and midwives perceptions of missed nursing care-A South Australian study. Collegian 2015; 22(4): 413-2O.

https://doi.org/10.1016/j.colegn.2014.09.001

48. Willis E, Harvey C, Thompson S, Pearson M, Meyer A. Work Intensification and Quality Assurance: Missed Nursing Care.J Nurs Care Qual. 2018;33(2): E10-E16.https://doi.org/10.1097/NCO.0000000000000277

49. Winsett RP, Rottet K, Schmit A, Wathen E, Wilson D, Missed Nursing Care Collaborative Group. Medical surgical nurses describe missed nursing care tasks-Evaluating our work environment. Appl. Nurs. Res. 2016; 32: 128-33. https://doi.org/10.1016/j.apnr.2016.06.006

50. Harvey C, Thompson S, Pearson M, Willis E, Toffoli L. Missed nursing care as an 'art form': the contradictions of nurses as carers. Nurs Inq. 2017; 24(3). https://doi.org/10.1111/nin.12180

51. Srulovici E, Drach-Zahavy A. Nurses' personal and ward accountability and missed nursing care: A cross-sectional study. Int J Nurs Stud. 2017;75:163-71. https://doi.org/10.1016/j.jinurstu.2017.08.003

52. Jangland Eva, Teodorsson T, Molander K, Muntlin Athlin $\AA$. Inadequate environment, resources and values lead to missed nursing care: A focused ethnographic study on the surgical ward using the Fundamentals of Care framework. J. Clin Nurs. 2018;27(11-12):2311-21.

https://doi.org/10.1111/jocn.14095 
53. Willis E, Carryer J, Harvey C, Pearson M, Henderson J. Austerity, new public management and missed nursing care in Australia and New Zealand. J Adv Nurs. 2017; 73(12): 3102-10.

https://doi.org/10.1111/jan.13380

54. Vryonides S, Papastavrou E, Charalambous A, Andreou P, Eleftheriou C, Merkouris A. Ethical climate and missed nursing care in cancer care units. Nurs Ethics 2018; 25(6): 707-23.

https://doi.org/10.1177/og69733016664979

55. Kalisch, BJ, Lee KH. Congruence of perceptions among nursing leaders and staff regarding missed nursing care and teamwork. J Nurs Adm. 2012; 42(10): 473-7.

https://doi.org/10.1097/NNA.obo13e31826a1fa4

56. Kalisch BJ, Xie B, Ronis DL. Train-the-trainer intervention to increase nursing teamwork and decrease missed nursing care in acute care patient units. Nurs Res. 2013; 62(6):405-13.

https://doi.org/10.1097/NNR.obo13e3182a7a15d

57. Tubbs-Cooley HL, Pickler RH, Mark BA, Carle AC. A research protocol for testing relationships between nurse workload, missed nursing care and neonatal outcomes: the neonatal nursing care quality study. J Adv Nurs. 2015; 71 (3): 632-41. https://doi.org/10.1111/jan.12507

58. Zúñiga F, Ausserhofer D, Hamers JP, Engberg S, Simon M, Schwendimann R. The relationship of staffing and work environment with implicit rationing of nursing care in Swiss nursing homes-a cross-sectional study.Int J Nurs Stud. 2015; 52(9):1463-74. https://doi.org/10.1016/j.ijnurstu.2015.05.005

59. Bagnasco A, Timmins F, de Vries JMA, Aleo G, Zanini M, Catania G, et al. Understanding and addressing missed care in clinical placements-Implications for nursing students and nurse educators. Nurs Educ Today 2017; 56:1-5. https://doi.org/10.1016/j.nedt.2017.05.015

6o. Ball JE, Griffiths P, Rafferty AM, Lindqvist R, Murrells T, Tishelman C. A cross-sectional study of 'care left undone'on nursing shifts in hospitals. J. Adv Nurs. 2016; 72(9): 2086-97. https://doi.org/10.1111/jan.12976

61. Dehghan-Nayeri N, Ghaffari F, Shali M. Exploring Iranian nurses' experiences of missed nursing care: a qualitative study: a threat to patient and nurses' health. Med J Islam Repub Iran. 2015; 29:276. 